\title{
Retinal ganglion cell loss in kinesin-1 cargo Alcadein a deficient mice
}

\author{
Yuki Nakano ${ }^{1}$, Kazuyuki Hirooka², Yoichi Chiba ${ }^{3}$, Masaki Ueno ${ }^{3}$, Daiki Ojima ${ }^{4}$, Md Razib Hossain ${ }^{4}$, Hiroo Takahashi ${ }^{4}$, \\ Tohru Yamamoto id ${ }^{4}$ and Yoshiaki Kiuchi ${ }^{2}$
}

\begin{abstract}
Maintenance of retinal ganglion cells (RGCs) activity is relied on axonal transport conveying materials required for their survival such as neurotrophic factors. Kinesin-1 undergoes anterograde transport in axons, and Alcadein a (Alca; also called calsyntenin-1) is a major cargo adaptor protein that can drive kinesin-1 to transport vesicles containing Alca. The long-term effects of Alca-deficiency on retinal morphology and survival of RGCs during postnatal development were examined in Alca knockout mice. At 1.5, 3, 6, and 15 months postnatal, the number of retrogradely labeled RGCs was determined in flat-mounted retinas of Alca-deficient and wild-type mice. Retinal damage was assessed histologically by determining the retinal thickness. Intraocular pressure (IOP) was measured with a Tonolab tonometer. At 1.5 months postnatal, the number of retrogradely labeled RGCs was not different between wild-type and Alcadeficient mice. However, at 3, 6, and 15 months postnatal, the number of RGCs was significantly lower in Alca deficient mice than those of wild-type mice $\left(143 \pm 41.1 \mathrm{cells} / \mathrm{mm}^{2}\right.$ vs. $208 \pm 28.4$ cells $/ \mathrm{mm}^{2}$, respectively, at 3 months; $\left.P<0.01\right)$. No differences were seen in retinal thickness or IOP between the two types of mice at any postnatal age. Alcadeficient mice showed spontaneous loss of RGCs but no elevation in IOP. These mice mimic normal-tension glaucoma and will be useful for investigating the mechanism of neurodegeneration in this disorder and for developing treatments for RGC loss that does not involve changes in IOP.
\end{abstract}

\section{Introduction}

Glaucoma involves axon pathology of retinal ganglion cells (RGCs) and is more commonly seen in older people and those with elevated intraocular pressure $(\mathrm{IOP})^{1}$. However, some patients exhibit disease progression even when their IOP is normal or controlled with medication. The mechanism of neurodegeneration in glaucoma is not clear. Multiple stimuli induce neuronal apoptosis, which may be reversed by neurotrophic factors that promote neuronal development and survival ${ }^{2}$. RGC survival is mediated by brain-derived neurotrophic factor (BDNF), nerve growth factor (NGF), ciliary neurotrophic factor (CNTF), and glial cell line-derived neurotrophic factor

\footnotetext{
Correspondence: Kazuyuki Hirooka (khirooka9@gmail.com)

'Department of Ophthalmology, Kagawa University Faculty of Medicine,

Kagawa, Japan

2Department of Ophthalmology and Visual Science, Graduate School of

Biomedical Sciences, Hiroshima University, Hiroshima, Japan

Full list of author information is available at the end of the article.

Edited by N. Bazan
}

$(\mathrm{GDNF})^{3}$. Intact axonal transport is prerequisite for transducing such trophic signals, and impaired axonal transport is recognized as an initial pathological event commonly observed in neurodegenerative disorders ${ }^{4}$. In various glaucoma models, deficits in axonal transport were observed after elevation of $\mathrm{IOP}^{5}$, however, it is still unclear whether attenuation of axonal transport could be a cause for RGC degeneration.

Kinesin-1 is a major molecular motor employed for fast anterograde axonal transport. Kinesin-1 is composed of two heavy chains (KIF5) and two light chains (KLC); KIF5 contains motor activity, and KLCs associate with KIF5 motors. Kinesin-1 is kept inactive in a cell by maintaining auto-inhibited state, which can be released by simultaneous interaction of cargo molecules with KIF5 tail domain and $\mathrm{KLC}^{6}$. Alcadein $\alpha$ (Alco; also called calsyntenin-1) is a member of neuronal type I single-pass transmembrane protein Alcadeins (also called calsyntenins), whose primary structures are evolutionarily 
conserved $^{7,8}$. Alco directly binds to kinesin light chain (KLC) through its C-terminal cytoplasmic region and is transported by kinesin- $1^{9}$. Furthermore, Alc $\alpha$ indeed activates kinesin-1 by itself; Alc $\alpha$ can release the autoinhibited state of kinesin- 1 by itself only through the $\sim 10$ amino acids length $\mathrm{W}$-acidic motif structure in the $\mathrm{C}$ terminal cytoplasmic region of Alca, forcing kinesin- 1 to be activated for anterogradely transporting Alc $\alpha$-containing vesicles ${ }^{10,11}$. It means that Alc $\alpha$-containing vesicles are transported by kinesin-1 in priority to another vesicles, suggesting that Alco would play an important role in transporting molecules that have to be preferentially delivered. These characteristic features of Alc $\alpha$ in axonal transport prompted us that Alc $\alpha$ deficiency could adversely affect homeostasis of neurons. In fact, in vitro studies showed that attenuation of Alco function by introducing dominant-negative Alc $\alpha$ or siRNA into cultured cells reduces axonal transport of amyloid precursor protein (APP) to increase cytotoxic $A \beta$ generation $^{9,12}$, and recent in vivo study showed that Alcodeficient mice eventually exhibited augmentation of amyloidogenic processing of endogeneous APP sufficient to lead pathologic feature of Alzheimer's disease such as amyloid plaque formation ${ }^{13}$. Given that APP is not the sole protein of which transport is affected by Alca, these observations collectively suggest that Alco-deficiency might induce vulnerability of neurons by attenuating efficient traffic of necessary factors, which could finally lead to their degeneration in particular neurons.

Here we found and report progressive loss of RGCs in Alco-deficient mice. We examined the long-term effect of Alca-deficiency on retinal morphology and discuss on the role of Alca in survival of RGCs during postnatal development.

\section{Materials and methods Experimental animals}

All animal investigations were performed according to the guidelines for animal experimentation of the Kagawa University Faculty of Medicine and adhered to the ARVO Statement for the Use of Animals in Ophthalmic and Vision Research. The mice were kept in a standard animal room with a 12-h light-dark cycle and free access to food and water. Generation of Alca knockout mice was described elsewhere ${ }^{13}$. Briefly, mouse genomic DNA including the first exon of CLSTN1 (the gene encoding Alca) was obtained from a C57BL/6 BAC clone (Invitrogen) and used to prepare a targeting vector. The vector was constructed by replacing the coding sequence with a LacZ-pA-PGK-Neo-pA cassette from the DT-A/LacZ/Neo plasmid; the vector was then electroporated into TT2 embryonic stem cells ${ }^{14}$. PCR with the following primers was performed to identify successful recombinants: $5^{\prime}$-A CCGCTTCCTCGTGCTTTACGGTATC- $3^{\prime}$ and $5^{\prime}$-T
AAGAACCTATTTAACAGGGGCTAGC-3'. Knockout mice were backcrossed to the C57BL/6 background for more than 10 generations. The PGK-Neo region of the cassette was removed by crossing these mice to transgenic C57BL/6 mice ubiquitously expressing flippase ${ }^{15}$. The wild-type allele and floxed $L a c Z-p A-p A$ allele were identified with PCR using the following primers: $5^{\prime}-C$ GGGGTCTGGGCCGCGCGAGGTAA- $3^{\prime}$ for wild type (416 bp); $5^{\prime}$-CGGGGTCTGGGCCGCGCGAGGTAA-3' and $5^{\prime}$-GCTGGCTGCCATGAACAAAGGTTGG- $3^{\prime}$ for LacZ-pA-pA (1224 bp).

\section{Histology}

The mice of indicated age and genotypes were sacrificed following an intraperitoneal overdose of pentobarbital, and eyes were enucleated immediately and fixed in $4 \%$ paraformaldehyde at room temperature. Next, the anterior segments, including the lens, were removed. The posterior eyecups were embedded in paraffin, and $5-\mu \mathrm{m}$ thick sections, which contained the full length of the eyecup from the superior to inferior surface along the vertical meridian through the optic nerve head, were cut using a microtome. Each eye was mounted on a glass slide coated with silane and stained with hematoxylin and eosin. The thickness of the sclera was measured to confirm that the sections were not oblique.

Images were taken of five randomly selected sections per eye, within $0.5 \mathrm{~mm}$ of the optic disc. One investigator blinded to the genotype of the mice performed all light microscopic assessments (magnification; $10 \times 100$; Olympus BX-51, Olympus Inc., Tokyo, Japan) and determined the thickness of the inner plexiform layer (IPL), inner nuclear layer (INL), outer plexiform layer (OPL), and outer nuclear layer (ONL).

In situ hybridization of Alc $\alpha$ was performed essentially as described ${ }^{16}$. Briefly, fixed and cryoprotected eyes were embedded in OCT compound (Sakura Finetech, Tokyo, Japan) and sectioned into serial 20- $\mu \mathrm{m}$ coronal sections on a CM3000 cryostat (Leica Microsystems, Wetzler, Germany). The resultant sections were post-fixed, washed three times with PBS, and incubated in $1 \mu \mathrm{g} / \mathrm{ml}$ Proteinase $\mathrm{K}$ (Roche Applied Science) in $6.25 \mathrm{mM}$ EDTA pH 8.0 (Dojindo Laboratories, Kumamoto, Japan) and $50 \mathrm{mM}$ Tris pH 7.5 (Wako Pure Chemical Industries, Osaka, Japan) at RT for $5 \mathrm{~min}$. The sections were re-fixed, washed with PBS, and acetylated in $1.33 \%$ triethanol amine (Sigma-Aldrich; St. Louis, MO), $0.75 \%$ acetic anhydride solution (Wako Pure Chemical Industries) at RT for $10 \mathrm{~min}$. The acetylated sections were washed with PBS and incubated in hybridization buffer ( $50 \%$ formamide (Sigma-Aldrich), $0.25 \mathrm{mg} / \mathrm{ml}$ Yeast RNA (Sigma-Aldrich), $0.5 \mathrm{mg} / \mathrm{ml}$ herring sperm DNA (Roche Applied Science), $5 x$ Denhard's (Sigma-Aldrich), 5x SSC $(0.75 \mathrm{M} \mathrm{NaCl}$, $75 \mathrm{mM}$ sodium citrate, $\mathrm{pH} 7.0)$ ) at $\mathrm{RT}$ for $2 \mathrm{~h}$, then with 
digoxigenin-labeled mouse Alc $\alpha$ cRNA probe in hybridization buffer at $72^{\circ} \mathrm{C}$ for $16 \mathrm{~h}$. The hybridized sections were washed in $5 \mathrm{x} \mathrm{SSC}$ at $72^{\circ} \mathrm{C}$ for $10 \mathrm{~min}$ and then in $0.2 \mathrm{x}$ SSC for $1 \mathrm{~h}$. The washed sections were incubated with 10\% heat-inactivated goat serum (Roche Applied Science) in $100 \mathrm{mM}$ Tris $\mathrm{pH} 7.5,0.15 \mathrm{M} \mathrm{NaCl}$ solution at RT for $1 \mathrm{~h}$, followed by incubation with alkaline phosphatase-conjugated anti-digoxigenin antibody (Roche Applied Science) in the same solution at $4{ }^{\circ} \mathrm{C}$ overnight. The sections were washed with $100 \mathrm{mM}$ Tris $\mathrm{pH} 7.5,0.15 \mathrm{M} \mathrm{NaCl}$ solution, then with $100 \mathrm{mM}$ Tris $\mathrm{pH}$ 9.5, $0.1 \mathrm{M} \mathrm{NaCl}, 50 \mathrm{mM} \mathrm{MgCl}_{2}$ solution, followed by incubation with NBT/BCIP (Roche Applied Science) in the same solution containing $0.24 \mathrm{mg} / \mathrm{mL}$ levamisole (Sigma-Aldrich) at RT in the dark. The reaction was stopped by immersing the sections in PBS-5 mM EDTA.

To detect Alco in RGCs by immunohistochemistry, anti-Alc $\alpha$ antibody ${ }^{9,17}$ was used and visualized by donkey secondary antibodies (Jackson Immuno Research Laboratories, West Grove, PA) as described ${ }^{16}$. After washing the sections with PBS, the slides were mounted with Shandon Immu-Mount (Cat \#9990402; Thermo Fisher Scientific, Waltham, MA) and observed by fluorescence microscopy with $\times 20$ objective and $\times 10$ eyepiece lens followed by merging respective images (BZ-9000; Keyence, Osaka, Japan).

\section{Western blot analysis}

To detect Alco in RGCs by western blotting, isolated retinal tissues were homogenized in RIPA buffer containing 1\% SDS and protease inhibitor cocktail $(5 \mu \mathrm{g} / \mathrm{ml}$ chymostatin, $5 \mu \mathrm{g} / \mathrm{ml}$ leupeptin, and $5 \mu \mathrm{g} / \mathrm{ml}$ pepstatin), and centrifuged $\left(15,000 \mathrm{rpm}\right.$ for $15 \mathrm{~min}$ at $\left.4{ }^{\circ} \mathrm{C}\right)$. The supernatants were used for western blot analysis as described ${ }^{16}$. Briefly, equal amounts of total protein $(1 \mu \mathrm{g})$ were loaded for each sample and run on a $12 \%$ polyacrylamide gel. The proteins were then transferred onto a PVDF membrane (Immobilon-P membrane, Millipore, Burlington, MA) using a wet tank system (BIO CRAFT, Tokyo, Japan). The membranes were blocked for $30 \mathrm{~min}$ at $\mathrm{RT}$ in $5 \%$ skim milk in TTBS $(20 \mathrm{mM}$ Tirs- $\mathrm{HCl} \mathrm{pH} 7.5$, $150 \mathrm{mM} \mathrm{NaCl}, 0.005 \%$ Tween-20, $5 \mathrm{mM} \mathrm{CaCl} 2$ ). After washing with TTBS, the membranes were incubated with the primary antibodies diluted in TTBS overnight at $4{ }^{\circ} \mathrm{C}$. After washing with TTBS, the membranes were treated with horseradish peroxidase (HRP)-conjugated secondary antibodies in TTBS for $1 \mathrm{~h}$ at RT. The following antibodies were used; anti-Alc $\alpha$ (guinea pig, 1:5000) ${ }^{9}$, anti- $\alpha$ tubulin (mouse, 1:5000, Abcam, Cambridge UK), HRPconjugated anti-guinea pig IgG (donkey, 1:15000, Jackson Immuno Research Laboratories), and HRP-conjugated anti-mouse IgG (sheep, 1:15000, GE Healthcare, Chicago, IL). Immunoreactive bands were detected by ECL Western Blotting Detection System (GE Healthcare), and captured with luminescent image analyzer (ImageQuant LAS 4010, GE Healthcare).

\section{Retrograde labeling of RGCs}

Mice were anesthetized with intraperitoneal injection of $0.75 \mathrm{mg} / \mathrm{kg}$ medetomidine, $4.0 \mathrm{mg} / \mathrm{kg}$ midazolam, and $5.0 \mathrm{mg} / \mathrm{kg}$ butorphanol before surgical procedures. The skull was exposed and kept clean and dry. Bregma was marked, and then a small window $(2.0 \mathrm{~mm}$ deep from the surface of the skull; $2.92 \mathrm{~mm}$ behind the bregma on the anteroposterior axis; $0.5 \mathrm{~mm}$ lateral to the midline) was drilled into the skull in both hemispheres. Using a Hamilton syringe (Hamilton Co., Reno, NV), $1.0 \mu$ l of $3 \%$ Fast Blue (Polysciences Inc., Warrington, PA) was slowly injected into the superior colliculi on both sides. The skin over the wound was sutured, and antibiotic ointment was applied. Mice were sacrificed 7 days later.

\section{Tissue preparation and assessment of RGC survival}

One week after Fast Blue injection, mice were sacrificed following an overdose of pentobarbital. RGC density was assessed in whole, flat-mounted retinas. Eyes were enucleated and fixed in $4 \%$ paraformaldehyde for $10 \mathrm{~h}$ at room temperature. The posterior eyecups were left in place after the anterior segments were removed. Next, four radial cuts were made in the periphery of each eyecup, and the retina was carefully removed from the retinal pigment epithelium. The retina was removed from other underlying structures, flattened by making four radial cuts, and mounted on a gelatin-coated glass slide. Labeled RGCs were visualized with fluorescence microscopy (Olympus BX-51/DP-72, Olympus, Tokyo, Japan) and an ultraviolet filter (excitation filter, $330-385 \mathrm{~nm}$; barrier filter, $420 \mathrm{~nm}$ ). RGCs were counted in 12 microscopic fields of the retina in two regions per quadrant at two different eccentricities from the optic disc: $0.3-0.8 \mathrm{~mm}$ (central) and $1.2-1.7 \mathrm{~mm}$ (peripheral). Image $J$ software (Version 1.51a, Wayne Rasband National Institutes of Health, USA) was used to determine the total number of RGCs per eye.

\section{Immunohistochemistry for Brn-3a}

Eyes were enucleated at 3, 8, and 15 months after birth, fixed in $4 \%$ paraformaldehyde, and embedded in paraffin. Retinal sections $(5 \mu \mathrm{m})$ were rinsed twice in $100 \%$ ethanol for $5 \mathrm{~min}$ each, and then rinsed for $3 \mathrm{~min}$ each with 95\% ethanol and then $90 \%$ ethanol. The sections were rinsed in phosphate-buffered saline (PBS, pH 7.4) three times for 10 min each and then incubated in $0.3 \%$ Triton X-100 in PBS for $1 \mathrm{~h}$. After further washing with PBS (three times for $10 \mathrm{~min}$ each), sections were blocked in 3\% normal horse serum and $1 \%$ bovine serum albumin (BSA) in PBS for $1 \mathrm{~h}$. Sections were incubated overnight at $4{ }^{\circ} \mathrm{C}$ in the primary antibody, rabbit polyclonal antibody against human Brn-3a (3:100; Catalogue Number: AB5945, 
Merck KGaA, Darmstadt, Germany) in 3\% BSA in PBS. Sections were washed three times for $5 \mathrm{~min}$ each in PBS and then incubated at room temperature for $30 \mathrm{~min}$ in Histofine Simple Stain MAX PO R (Nichirei Bioscience Inc., Tokyo, Japan) as the secondary antibody. Sections were washed three times for 5 min each in PBS and then incubated for $5 \mathrm{~min}$ at room temperature in Histofine DAB (Nichirei Bioscience Inc.) to allow color development. Sections were washed for $5 \mathrm{~min}$ in water, counterstained for $1 \mathrm{~min}$ at room temperature in Mayer's Hematoxylin Solution, and washed for $5 \mathrm{~min}$ in water. Images of stained sections were acquired using $\times 40$ objective lenses (DXM 1200; Nikon, Tokyo, Japan). Adobe PhotoShop v. 5.0 was used to adjust the brightness and contrast of the images.

\section{IOP measurement}

Mice were anesthetized by intraperitoneal injection of $0.75 \mathrm{mg} / \mathrm{kg}$ medetomidine, $4.0 \mathrm{mg} / \mathrm{kg}$ midazolam, and $5.0 \mathrm{mg} / \mathrm{kg}$ butorphanol, and then IOP was measured with a TonoLab tonometer (TioLat, Inc., Helsinki, Finland). We calculated the mean of six readings using the optimal variability grade.

\section{Statistical analysis}

All data are shown as the mean \pm standard error of the mean (SEM). All data were in a normal distribution, and variance was similar between the groups that are being statistically compared. Data were analyzed with an independent Student's $t$-test or Student's $t$-test, as appropriate. There were no exclusion criteria. Statistical analyses were performed with SPSS version 19.0 (SPSS Inc., Chicago, IL), and $P<0.05$ was considered statistically significant. Randomization, blinding, and sample size estimation tests were not done for our animal studies.

\section{Results}

\section{RGC loss in Alca-deficient mice}

We first examined the gross anatomy of 6-, 12-, and 24month-old Alc $\alpha$-deficient mice brains. Despite of above anticipation, including progressed $\mathrm{AD}$ pathogenesis of APP23 transgenic mice expressing human APP in Alcodeficient background ${ }^{13}$ (APP23 was utilized for detecting amyloid plaque formation, since mouse $\mathrm{A} \beta$ does not make aggregation that is prerequisite for the plaque formation), we did not find apparent anatomical or pathological alteration between wild-type and Alc $\alpha$-deficient mice brains in these age (data not shown). Then we switched our investigation to afferent neurons including RGCs.

We verified expression of Alca in retina of adult mice with in situ hybridization, immunohistochemistry, and western blotting. Alc $\alpha$ is expressed in retina including RGCs, which is not detected in Alc $\alpha$ knockout mice, confirming their Alc $\alpha$-deficiency in the retina (Fig. 1).
We then examined if there is difference in RGCs between 15-month-old wild-type and Alco-deficient mice by retrogradely labelling RGCs with Fast Blue bilaterally injected into their superior colliculi. Seven days after injection, numbers of labeled RGCs were counted (Fig. 2a). The numbers of RGCs in Alc $\alpha$-deficient mice were shown as percentages compared with those of wildtype mice. Statistically significant difference was observed in their numbers of RGCs: numbers of labelled RGCs were significantly lower than those of wild-type mice both in the central $(69.8 \pm 8.3 \%, P<0.01, n=8)$ and peripheral retina $(58.3 \pm 8.3 \%, P<0.01, n=8)$ (Fig. $2 b)$, indicating that Alco-deficiency caused loss of RGCs at this age.

We next verified the onset of the observed RGC loss. We examined the numbers of RGCs in Alco-deficient mice at 1.5, 3, and 6 months old with the same retrograde labelling. RGC loss was not observed in 1.5month-old Alc $\alpha$-deficient mice, indicating that generation and maintenance of RGCs are unaltered until adolescent stage. However, significant RGC loss was observed at 3-month-old Alco-deficient mice: numbers of labelled RGCs were significantly lower than those of wild-type mice both in the central $(73.0 \pm 8.4 \%, P=0.01$, $n=10)$ and peripheral retina $(68.0 \pm 6.5 \%, P<0.01, n=$ 10) (Fig. 3). Statistically significant loss of RGCs was also observed at 6 months of age both in the central $(79.3 \pm$ $5.4 \%, P<0.01, n=7)$ and peripheral retina $(73.0 \pm 4.1 \%$, $P<0.01, n=7$ ) (Fig. 3). These observations suggest that Alco-deficiency-induced RGC loss is not due to developmental defect but due to deficit in maintaining RGC activity.

\section{Loss of $\mathrm{Brn3a}^{+} \mathrm{RGCs}$ in Alca-deficient mice}

Alc $\alpha$ possesses privileged activity that drives kinesin-1 by itself, and it is highly plausible that Alco-deficiency should affect some aspects of axonal transport. Thus, in theory, it is hard to exclude the possibility that Alc $\alpha$ deficiency might affect efficiency of retrograde labelling of RGCs with Fast Blue, even though fairly enough time (7 days) was allowed to label the cells, and no significant difference was observed in the numbers of labelled RGCs of 1.5-month-old mice. To verify the possibility, we directly counted the numbers of Brn3a-expressing RGCs in the retinal sections of wild-type and Alc $\alpha$-deficient mice (Fig. 4a) at 3, 8, and 15 months of age (Fig. 4b, c). Brn3a is a transcription factor that is selectively expressed in great majority of RGCs in rodent retina ${ }^{18,19}$. Brn3a-positive RGCs were significantly decreased in Alco-deficient mice at 3 months of age (central retina: $68.9 \pm 6.5 \%, P=0.01, n=5$; peripheral retina: $56.3 \pm$ $4.7 \%, P<0.01, n=5), 8$ months of age (central retina: $81.5 \pm 5.6 \%, P=0.01$, peripheral retina: $72.9 \pm 3.8 \%, P<$ $0.01, n=9$ ), and 15 months of age (central retina: $70.9 \pm$ 8.1\%, $P<0.01$, peripheral retina: $64.7 \pm 9.3 \%, P<0.01$, 

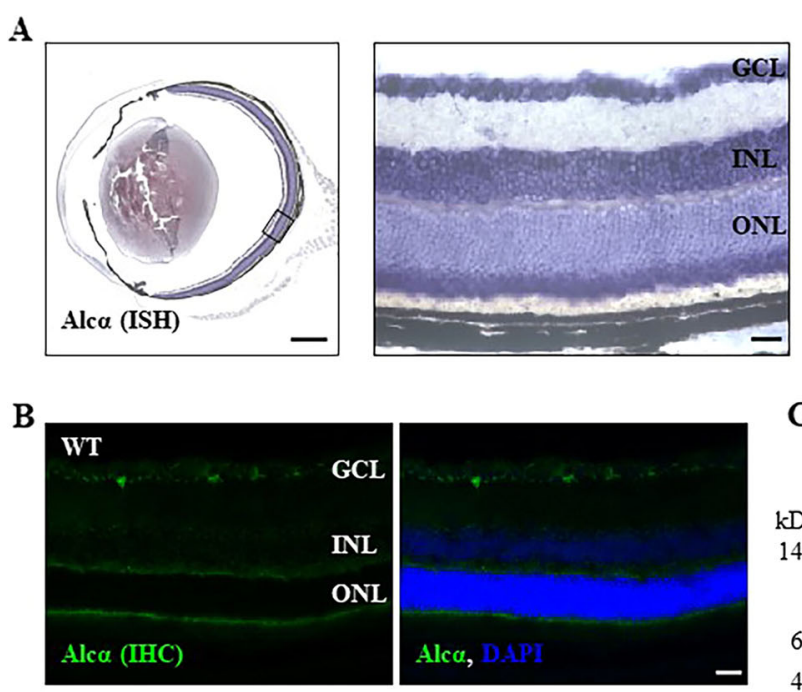

C
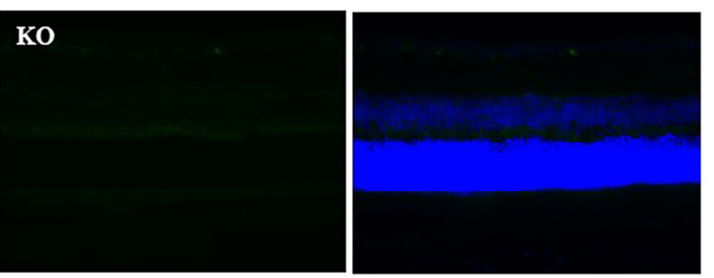

Fig. 1 Expression of Alca in the mouse retina. a Expression of Alca mRNA in an eye of 5-month-old wild-type mice was detected by in situ hybridization (ISH). The magnified image in the boxed area of the left side image is shown on the right side. Alca mRNA is readily detected in ganglion cells, cells in inner nuclear layer, and photoreceptors' inner segment. GCL: ganglion cell layer, INL: inner nuclear layer, ONL: outer nuclear layer. Scale bar, $500 \mu \mathrm{m}$. b Expression of Alca protein in an eye of 5-month-old wild-type (WT) and Alca-deficient (KO) mouse was detected by immunohistochemistry $(\mathrm{IHC})$. Alca protein is readily detected in ganglion cells, photoreceptors' inner segment, and outer peripheral layer. Alca protein is not detected in the eye of Alca-deficient mouse. Co-stained images with DAPI are shown on the right sides. GCL: ganglion cell layer, INL: inner nuclear layer, ONL: outer nuclear layer. Scale bar, 50 um. c Expression of Alca protein in a retina of 2-month-old wild-type (WT) and Alcadeficient $(\mathrm{KO})$ mouse was detected by western blotting. Alca protein is not detected in the retinal lysate $(1 \mu \mathrm{g})$ of Alca-deficient (KO) mouse.

$n=7)$, compared with those of wild-type mice, confirming that Alc $\alpha$-deficiency causes loss of RGCs.

\section{Thicknesses of other layers in retina were not significantly altered in Alca-deficient mice}

Alco is also expressed in other retinal cells (Fig. 1), of which deficiency might affect the maintenance of these cells. To verify the possibility, retinal layer thickness analysis was performed to check for degeneration of any of the other retinal neurons at the age of 15 months. The percent thickness in Alc $\alpha$-deficient mice compared with control mice showed no statistically significant difference: $96.5 \pm 7.2 \%(P=0.63)$ for the IPL, $91.4 \pm 5.6 \%(P=0.21)$ for the INL, $89.8 \pm 2.8 \%(P=0.06)$ for the OPL, and $91.4 \pm 5.6 \%(P=0.23)$ for the outer nuclear layer (Fig. 5$)$, suggesting that Alco-deficiency selectively affects survival of RGCs in retina.

\section{IOP was not altered in Alca-deficient mice}

To verify the effect of Alco on IOP, IOP of wild-type and Alc $\alpha$-deficient mice was measured at various ages.
We found no significant differences in IOP between wildtype and Alc $\alpha$-deficient mice at any age (Fig. 6) $(P=$ $0.07-0.37, n=6-20)$, indicating that RGC loss in Alc $\alpha-$ deficient mice was independent of IOP.

\section{Discussion}

Axonal transport plays a pivotal role in maintaining neuronal activity, and its deficit is one of common mechanisms underlying early-stage pathophysiology of various neurodegererative disorders. Alc $\alpha$ is an evolutionary conserved characteristic kinesin-1 cargo protein: that is not only transported by kinesin-1, but is also able to drive kinesin-1 to transport Alc $\alpha$-containing vesicles. We examined Alco-deficient mice in the context of neuroanatomy and degeneration, and found that RGCs were lost in these mice without elevation of IOP. Our studies added an evidence affirming importance of axonal transport on maintenance of RGCs, and further suggesting that Alco plays a role necessary for RGC survival and may be closely associated with normal-tension glaucoma (NTG) pathology. 


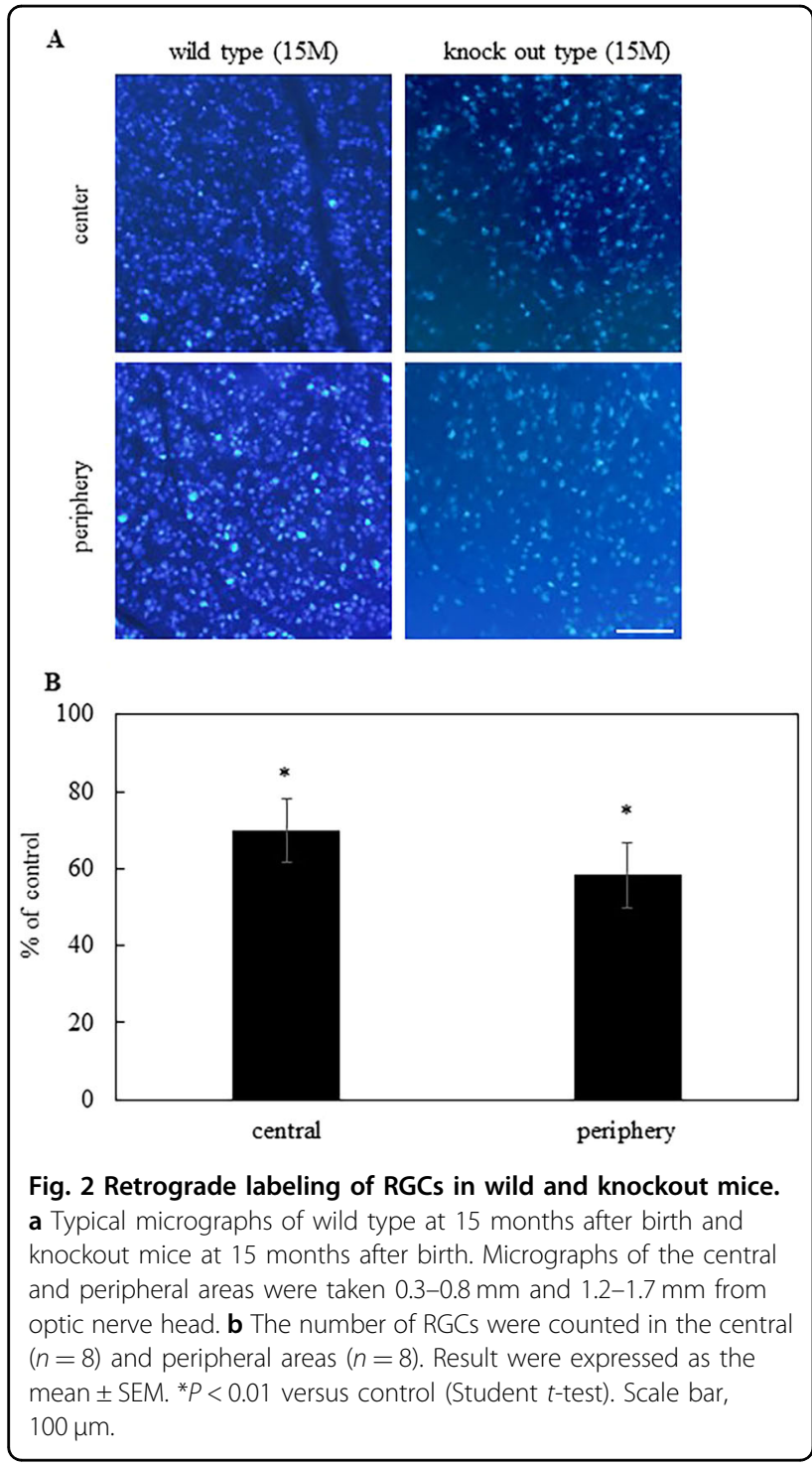

There are several possibilities how Alc $\alpha$ contributes RGC maintenance. Alco is involved in anterograde axonal transport, and deficiency of Alc $\alpha$ could perturb homeostatic regulation of axonal transport including retrograde transport; since motors and adaptors required for retrograde transport have to be delivered to nerve terminals once. Target-derived neurotrophic factors are essential for survival of neurons including RGC, and it would be plausible that inefficient delivery of such trophic factors from the targets might be involved in loss of RGCs in Alc $\alpha$-deficient mice. BDNF is reported as one of prominent target-derived trophic factor, of which administration protects RGCs from their death after nerve injury. We verified the amount of BDNF in the retina of Alc $\alpha$ deficient mice, and found no statistically significant difference (data not shown). Ineffective delivery of other neurotrophic factors such as CNTF, GDNF, and NGF

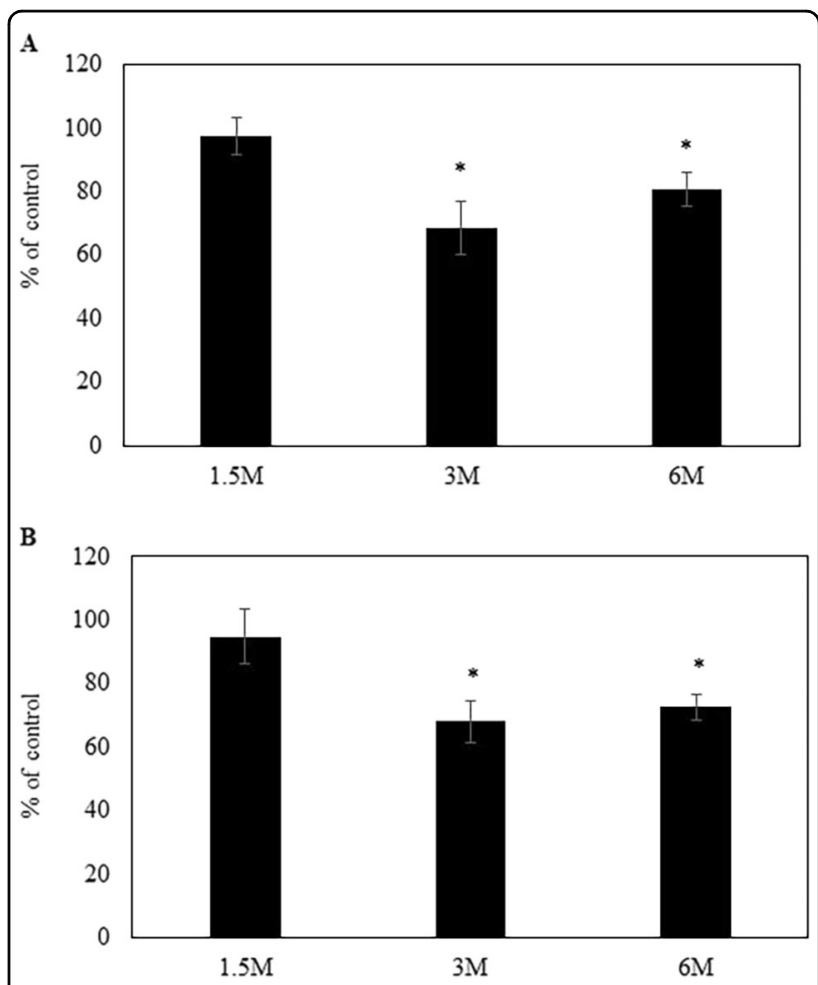

Fig. 3 The number of RGCs of knockout mice at $1.5(n=8), 3$ $(n=10)$, and $6(n=7)$ months after birth. a In the central retina, RGC was decreased 3 months after birth. $\mathbf{b}$ In the peripheral retina, reduction of number of RGCs was also detected at 3 months after birth. ${ }^{*} P<0.05$ versus control (Student $t$-test).

might contribute to the vulnerability of Alco-deficient RGC. Another possible candidate is molecules preferentially transported with Alc $\alpha$-containing vesicles. It was reported that axon guidance receptors Robo1 and Frizzled 3 are transported with Alco in developing chick spinal commissural neurons ${ }^{20}$. Frizzled is a Wnt receptor, and canonical Wnt signaling is reportedly transduced into a population of adult $\mathrm{RGCs}^{21}$. Alc $\alpha$-deficiency might cause inefficient transport of such molecules to reduce tolerance of RGCs to external stress leading loss of RGCs. Further investigation should be required for verifying these possibilities.

It has been shown that Alca is involved in regulation of amyloidogenic processing of APP: attenuation of Alc $\alpha$ reduces transport of APP to augment cytotoxic A $\beta$ production $^{9,12}$, and Alc $\alpha$-deficient mice exhibit enhanced amyloidogenic processing of APP to generate more A $\beta$ sufficient for exacerbating AD related pathogenesis such as amyloid plaque formation ${ }^{13}$. Alzheimer's disease is characterized by the death of hippocampal and cerebral cortical neurons due to oligomerized amyloid $\beta(A \beta)$ and tau protein in the brain ${ }^{22,23}$. Several studies have suggested a relationship between glaucoma and neurodegenerative diseases such as Alzheimer's disease ${ }^{24,25}$. 

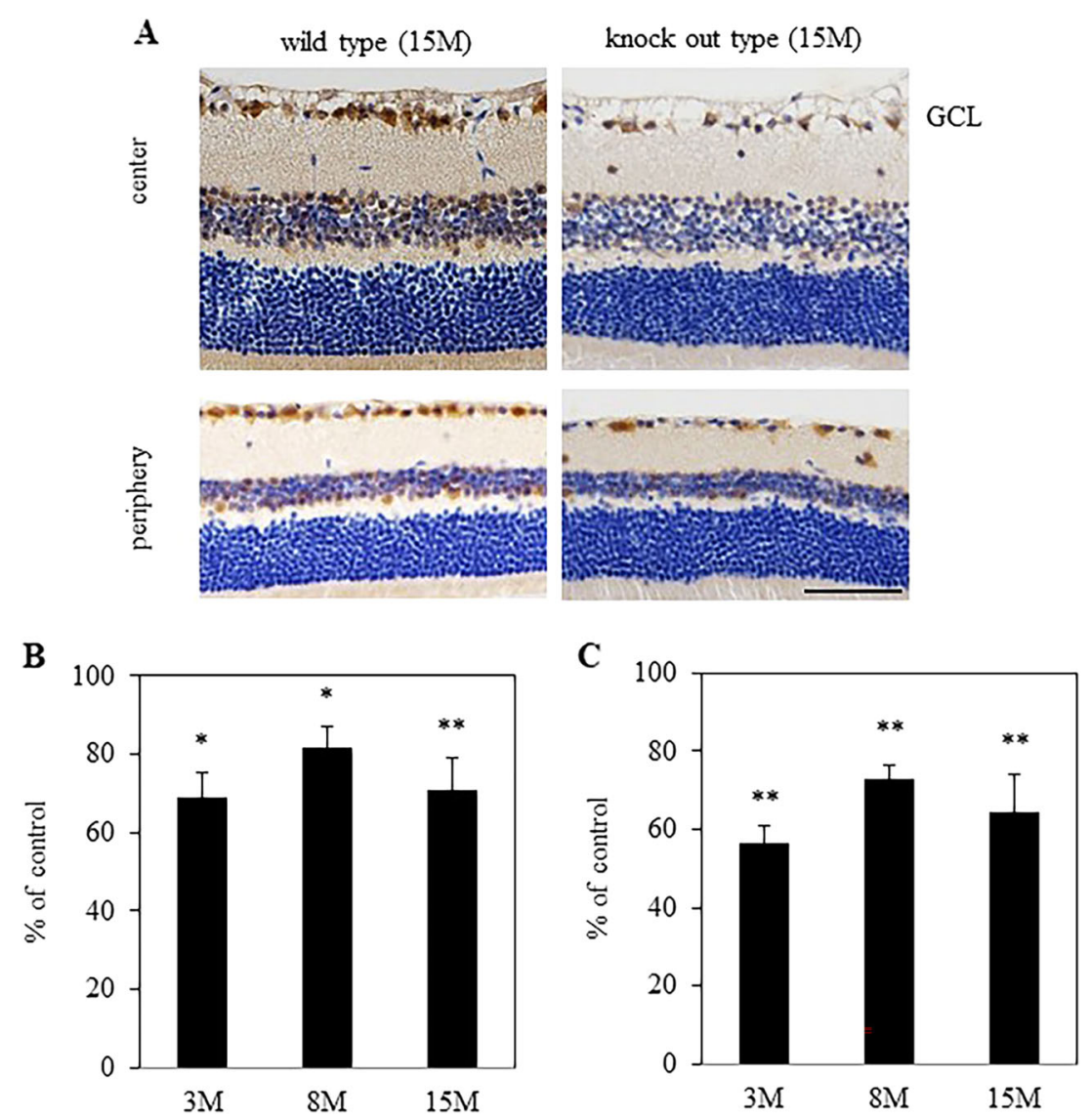

Fig. 4 Evaluating the number of RGCs with Brn-3a immunostaining. a Typical micrographs of Brn-3a immunostaining in wild-type and knockout type at 15 months after birth. Micrographs of the central and peripheral areas were taken $0.3-0.5 \mathrm{~mm}$ and $1.4-1.6 \mathrm{~mm}$ from optic nerve head. The number of RGCs were counted with Brn-3a immunostaining in the central (b) and peripheral (c) areas. Result were expressed as the mean \pm SEM. $3 \mathrm{M}$ : $n=5,8 \mathrm{M}: n=9,15 \mathrm{M}: n=7 .{ }^{*} P<0.05,{ }^{* *} P<0.01$ versus control (Student $t$-test). Scale bar, $50 \mu \mathrm{m}$.

Animal models have been used to test the relationship between $A \beta$ and glaucoma. Wang et al. ${ }^{26}$ reported that $A \beta$ is toxic to RGCs in vivo and showed that $A \beta$ is present adjacent to apoptotic RGCs, suggesting a possible role for $A \beta$ neurotoxicity in the development of RGC death in glaucoma. Kipfer-Kauer et al. ${ }^{27}$ reported caspase activation and abnormal APP processing in RGCs of rats with induced chronic ocular hypertension. It was recently reported that $A \beta$ peptides primarily inhibit glutamate reuptake to sensitize neurons for stimulations getting them more hyperactive ${ }^{28}$. RGC is also susceptible to excitotoxicity ${ }^{29}$, and such $\mathrm{A} \beta$-mediated hyperactivity might be a cause of RGC loss in Alco-deficient mice. Further investigation aimed for understanding the mechanisms of regulation of $\mathrm{A} \beta$ generation in the eye may become important step for understanding NTG pathogenesis.

Glaucoma is an age-related neurodegenerative disorder. In Alc $\alpha$-deficient mice, generation and maintenance of RGCs appeared normal until adolescent stage, suggesting that Alco-deficient mice exhibited no apparent effect on development and maturation, partly reflecting human glaucoma. Loss of RGCs was observed from 3 months of age, and the apparent ratio of lost RGCs looks rather constant ( $30 \%)$ along with age, although numbers of eyes exhibiting lower than mean minus 2 standard deviation. of wild-type mice seem to increase in older mice. It is obscure why Alco-deficient mice showed such step-wise loss of their RGC. One of possibilities would be that some populations of RGCs are more susceptible to circumstantial stress accumulated and/or become evident by the age. RGCs are not homogeneous but consist of $\sim 30$ subtypes, of which function and dendritic structures are different ${ }^{30}$. Some of these subtypes of RGCs may be more vulnerable than other subtypes in Alco-deficient condition. Given that Wnt signaling is transduced into a subpopulation of adult $\mathrm{RGCs}^{21}$, some of molecules involved in such trophic mechanism may be inefficiently transported without Alc $\alpha$, and the most labile subpopulations of RGCs to those deficiencies might become degenerated around those time points. It should not be also excluded that 15 months old may not be old enough, and more RGC loss might occur in much older mice. Further 

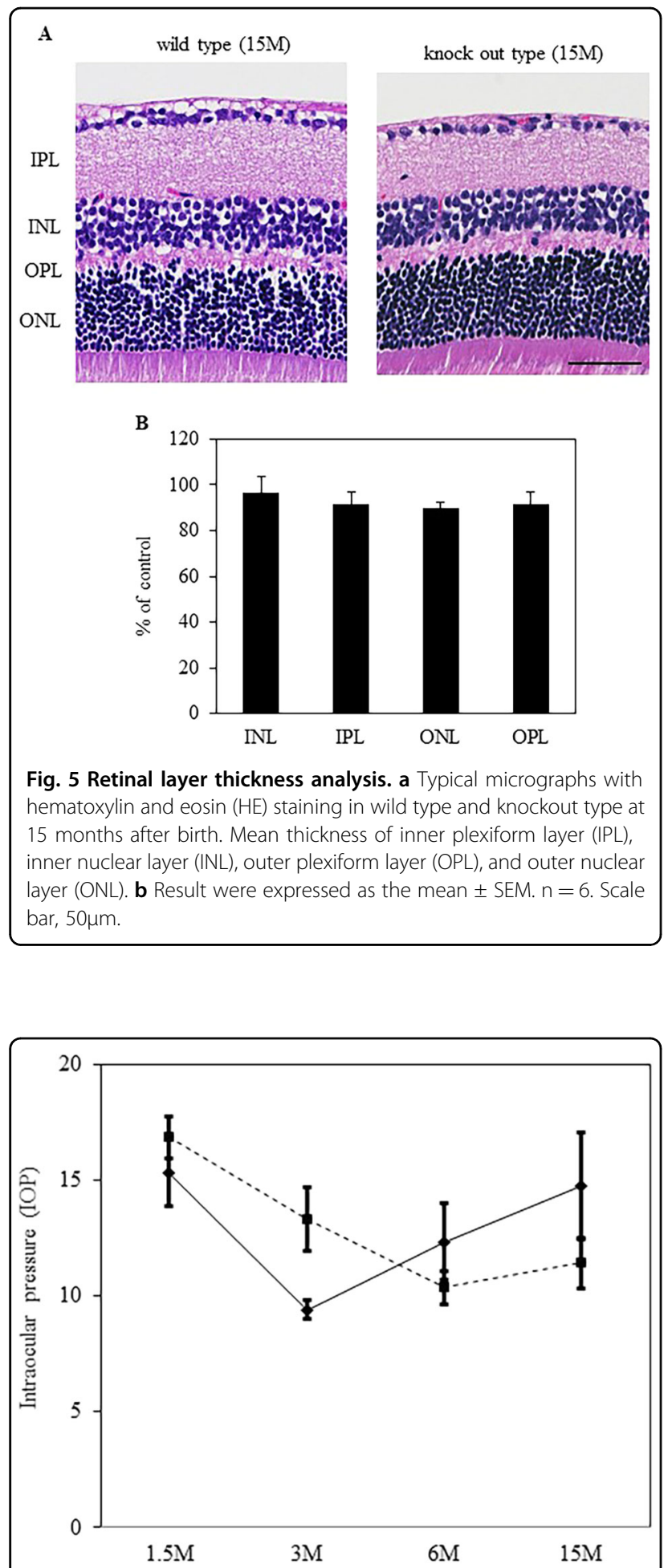

Fig. 6 Intraocular pressure (IOP) in wild type and knockout type. Result were expressed as the mean \pm SEM. There were no significantly differences in the IOP between wild-type and knockout mice. Wild type: broken line, knockout type: solid line. Wild type; $1.5 \mathrm{M}: n=10$, $3 \mathrm{M}: n=10,6 \mathrm{M}: n=20,15 \mathrm{M}: n=8$. Knockout type; $1.5 \mathrm{M}: n=8,3 \mathrm{M}$ : $n=10,6 \mathrm{M}: n=6,15 \mathrm{M}: n=6$. investigation including analyzing older mice would be required to verify the possibilities.

In conclusion, Alc $\alpha$-deficient adult mice showed RGC loss that was independent of elevated IOP. We believe that this animal model could be utilized as a tool for investigating the mechanisms of neurodegeneration in NTG and for developing treatments for IOP-independent RGC loss.

\section{Acknowledgements}

This work was supported by a Grant-in-Aid for Scientific Research from the Ministry of Education, Culture, Sports, Science, and Technology of Japan (26462689).

\section{Author details}

'Department of Ophthalmology, Kagawa University Faculty of Medicine, Kagawa, Japan. ${ }^{2}$ Department of Ophthalmology and Visual Science, Graduate School of Biomedical Sciences, Hiroshima University, Hiroshima, Japan. ${ }^{3}$ Department of Inflammation Pathology, Kagawa University Faculty of Medicine, Kagawa, Japan. ${ }^{4}$ Department of Molecular Neurobiology, Kagawa University Faculty of Medicine, Kagawa, Japan

\section{Conflict of interest}

The authors declare that they have no conflict of interest.

\section{Publisher's note}

Springer Nature remains neutral with regard to jurisdictional claims in published maps and institutional affiliations.

Received: 9 December 2019 Revised: 11 February 2020 Accepted: 20 February 2020

Published online: 03 March 2020

\section{References}

1. Calkins, D. J. Critical pathogenic events underlying progression of neurodegeneration in glaucoma. Prog. Retin Eye Res. 31, 702-719 (2012).

2. Micera, A. et al. Differential protein expression profiles in glaucomatous trabecular meshwork: an evaluation study on a small primary open angle glaucoma population. Adv. Ther. 33, 252-267 (2016).

3. Almasieh, M., Wilson, A. M., Morquette, B., Cueva, Vargas, J. L. \& Di Polo, A. The molecular basis of retinal ganglion cell death in glaucoma. Prog. Retin Eye Res. 31, 152-181 (2012).

4. Millecamps, S. \& Julien, J.P. Axonal transport deficits and neurodegenerative diseases. Nat. Rev. Neurosci. 14, 161-176 (2013).

5. Fahy, E. T., Chrysostomou, V. \& Crowston, J. G. Mini-review: impaired axonal transport and glaucoma. Curr. Eye Res. 41, 273-283 (2016).

6. Verhey, K. J., Kaul, N. \& Soppina, V. Kinesin assembly and movement in cells. Annu Rev. Biophys. 40, 267-288 (2011).

7. Hintsch, G. et al. The calsyntenins - a family of postsynaptic membrane proteins with distinct neuronal expression patterns. Mol. Cell Neurosci. 21, 393-409 (2002).

8. Araki, Y. et al. Novel cadherin-related membrane proteins, Alcadeins, enhance the X11-like protein mediated stabilization of amyloid $\beta$-protein precursor metabolism. J. Biol. Chem. 278, 49448-49458 (2003).

9. Araki, Y. et al. The novel cargo Alcadein induces vesicle association of kinesin-1 motor components and activates axonal transport. EMBO J. 26, 1475-1486 (2007).

10. Kawano, T. et al. A small peptide sequence is sufficient for initiating kinesin-1 activation through part of TPR region of KLC1. Traffic 13, 834-848 (2012).

11. Yip, Y. Y. et al. The light chains of kinesin-1 are autoinhibited. Proc. Natl Acad. Sci. USA 113, 2418-2423 (2016).

12. Vagnoni, A. et al. Calsyntenin-1 mediates axonal transport of the amyloid precursor protein and regulates $A \beta$ production. Hum. Mol. Genet. 21, 2845-2854 (2012).

13. Gotoh, N. et al. APP amyloidogenic processing is enhanced in the brains of Alcadein a-deficient mice. Under submission. 
14. Yagi, T. et al. A novel ES cell line, TT2, with high germline-differentiating potency. Anal. Biochem 214, 70-76 (1993).

15. Tian, E., Kimura, C., Takeda, N., Aizawa, S. \& Matsui, I. Otx2 is required to respond to signals from anterior neural ridge for forebrain specification. Dev. Biol. 242 204-223 (2002).

16. Ishikawa, T. et al. IgSF molecule MDGA1 is involved in radial migration and positioning of a subset of cortical upper-layer neurons. Dev. Dyn. 240, 96-107 (2011).

17. Maruta, C. et al. Constitutive cleavage of the single-pass transmembrane protein alcadeina prevents aberrant peripheral retention of Kinesin-1. PLoS ONE 7, e43058 (2012)

18. Quina, L. A. et al. Brn3a-expressing retinal ganglion cells project specifically to thalamocortical and collicular visual pathways. J. Neurosci. 25, 11595-11604 (2005).

19. Nadal-Nicolás, F. M. et al. Brn3a as a marker of retinal ganglion cells: qualitative and quantitative time course studies in naive and optic nerve-injured retinas. Invest Ophthalmol. Vis. Sci. 50, 3860-3868 (2009).

20. Alther, T. A., Domanitskaya, E. \& Stoeckli, E. T. Calsyntenin 1-mediated trafficking of axon guidance receptors regulates the switch in axonal responsiveness at a choice point. Development 143, 994-1004 (2016).

21. Liu, H., Thurig, S., Mohamed, O., Dufort, D. \& Wallace, V. A. Mapping canonical Wnt signaling in the developing and adult retina. Invest Ophthalmol. Vis. Sci. 47, 5088-5097 (2009).
22. Wyss-Coray, T. Inflammation in Alzheimer disease: driving force, bystander or beneficial response? Nat. Med. 12, 1005-1015 (2006).

23. Gupta, N., Fong, J., Ang, L. C. \& Yücel, Y. H. Retinal tau pathology in human glaucomas. Can. J. Ophthalmol. 43, 53-60 (2008).

24. Tsolaki, F. et al. Alzheimer's disease and primary open-angle glaucoma: is there a connection? Clin. Ophthalmol. 5, 887-890 (2011).

25. Nucci, C. et al. Links among glaucoma, neurodegenerative, and vascular diseases of the central nervous system. Prog. Brain Res. 221, 49-65 (2015).

26. Wang, J. et al. Development and expression of amyloid- $\beta$ peptide 42 in retinal ganglion cells in rat. Anat. Rec. 294, 1401-1405 (2011).

27. Kipfer-Kauer, A., McKinnon, S. J., Frueh, B. E. \& Goldblum, D. Distribution of amyloid precursor protein and amyloid-beta in ocular hypertensive C57BL/6 mouse eyes. Curr. Eye Res. 35, 828-834 (2010).

28. Zott, B. et al. A vicious cycle of $\beta$ amyloid-dependent neuronal hyperactivation. Science 365, 559-565 (2019).

29. Almasieh, M., Wilson, A. M., Morquette, B., Cueva, Vargas, J. L. \& Di Polo, A. The molecular basis of retinal ganglion cell death in glaucoma. Prog. Retin Eye Res. 31, 152-161 (2012)

30. Sane, J. R. \& Masland, R. H. The types of retinal ganglion cells: current status and implications for neuronal classification. Annu Rev. Neurosci. 38, 221-246 (2015). 